\title{
Intervención temprana en prematuros, una experiencia de trabajo
}

Avilés A.Clara,; Madariaga S. Patricia; Fuentes L. Patricia; et al

\begin{abstract}
" Resumen
Propósito: Aplicar técnicas de estimulación motora y neurosensorial global enfocadas a favorecer un adecuado desarrollo psicomotor en niños con antecedente de prematurez y menos de 1500 grs de peso al nacer. Metodología: Se trabajó con una muestra de 46 niños con antecedente de menos de 1500 grs de peso al nacer y edad gestacional entre 26 y 36 semanas, los cuales a su alta de la Unidad de Neonatología del Hospital Clínico Regional de Concepción fueron ingresados al Servicio de Medicina Física y Rehabilitación del mismo establecimiento, para su evaluación y seguimiento por parte de Kinesióloga y Terapeuta Ocupacional. Se aplicaron evaluaciones de desarrollo psicomotor y entregaron indicaciones a los padres sobre medidas de estimulación neurosensorial y motora en forma mensual hasta el octavo mes de edad corregida.
\end{abstract}

Resultados: Los resultados de las intervenciones, medidos a través de las evaluaciones efectuadas a los 12 meses de edad corregida, permiten concluir la efectividad y validez de las técnicas de estimulación aplicadas.

Conclusiones: Los resultados obtenidos permitirían verificar la validez de una intervención sistematizada, con el fin de detectar e intervenir oportunamente los casos de riesgo o retraso psicomotor que se registran en la población en estudio.

\begin{abstract}
Purpose: Apply techniques of global motor and neurosensory stimulation focus on supporting adequate psychomotor development in premature infants with antecedent of less than 1500 grs when they were born. Method: It was worked with a sample of 46 children with antecedent of less than 1500 grs when they were born and a gestative age between 26 and 36 weeks, which they certificate of discharge of the Neonatology Service of Hospital Clínico Regional in Concepción were entered to the Physical Medicine and Rehabilitation Service of the same institution, to be evaluated and followed up by psysiotherapist and occupational therapist. There were applied evaluations of the psychomotor development and gave indications to their parents about the measuren of motor and neurosensorial stimulation monthly until the eighth month corrected. Results: The intervention results, measured through evaluation made at 12 months of corrected age, allow us to conclude effectiveness and validity of the applied stimulation techniques.
\end{abstract}

Conclusions: Obtained results would allow us to verify the systematized intervention validity, with the purpose of detecting and interceding opportunely the cases of risk or psychomotor delay that are detected in the population under investigation.

Terapeuta Ocupacional, Licenciada en Ciencia de la Ocupación. Servicio de Medicina Física y Rehabilitación. Hospital Clínico Regional de Concepción. Kinesióloga, Servicio de Medicina Física y Rehabilitación. Hospital Clínico Regional de Concepción. Contacto > > Servicio de Medicina Física y Rehabilitación. Hospital Clínico Regional de Concepción 


\section{- Introducción}

En el campo de la salud están abundantemente estudiadas las consecuencias negativas que puede tener en el desarrollo de un niño la presencia de factores de riesgo biológico (enfermedades congénitas, lesiones perinatales, etc.), y la forma en que ello se potenciará si el niño se encuentra inserto en un medio deprimido socio-cultural y económicamente, que le impida recibir adecuado tratamiento médico, en forma oportuna y sistemática $\left.{ }^{(1}\right)$.

Un importante factor de riesgo lo constituye actualmente el nacimiento prematuro, ya que debido a los avances en la medicina perinatal ha aumentado la sobrevida de los niños prematuros de muy bajo peso. La Organización Mundial de la Salud define como prematuro ${ }^{(2)}$ a aquel recién nacido antes de las 37 semanas de embarazo cumplidas, lo que corresponde al $1 \%$ de los recién nacidos vivos. Dentro de este grupo, aquellos niños nacidos con 1500 grs de peso o menos corresponden al grupo que amerita las mayores atenciones ${ }^{(3)}$, por constituir el grupo en riesgo de presentar discapacidad en etapas posteriores de su vida. En Chile, diversos estudios ${ }^{(1)}$ muestran rangos entre 6,7 y $32 \%$ de déficits mayores en niños nacidos con menos de 1000 grs. Se define como déficits mayores a la parálisis cerebral, retardo mental, sordera neurosensorial, defectos visuales severos, hidrocefalia y convulsiones. También se describe la presencia de problemas intelectuales en la edad escolar y la adolescencia.

Fueron estos antecedentes los que en el año 1998, ante el creciente número de niños que actualmente nacen prematuros en nuestro país, y dado que en el Servicio de Salud Concepción no existían en ese momento políticas específicas sobre la evaluación del desarrollo psicomotor de estos niños ${ }^{(4)}$ motivaron al equipo de rehabilitación pediátrica del Servicio de Medicina Física y Rehabilitación del Hospital Clínico Regional de Concepción, a diseñar un programa destinado a prevenir la aparición de retraso psicomotor y estimular el normal desarrollo en estos niños, así como a proponer líneas de intervención desde nuestras disciplinas. Las Orientaciones Técnicas Para el Seguimiento de los Prematuros fueron propuestas por el Ministerio de Salud (MINSAL) en $1999^{(4)}$.

\section{- Marco Teórico}

Las más recientes investigaciones en el campo de la neurobiología han sido específicas para demostrar cómo las neuronas de la corteza cerebral en un niño pequeño se van interconectando, de acuerdo a un esquema determinado genéticamente y según van recibiendo estímulos del medio ambiente (plasticidad del sistema nervioso) ${ }^{(\underline{5})}$.

Las mismas investigaciones son enfáticas en establecer que de no recibir estímulos, las neuronas desarrollan menos interconexiones, e incluso pueden morir. Una parte importante relativa a la forma en que se interconectan las neuronas está genéticamente determinada, principalmente aquellas destinadas al control de la respiración, ritmo cardíaco, regulación de la temperatura corporal o reflejos.

Sin embargo, otra porción muy significativa depende de los estímulos del medio ambiente para su interconección. Las mismas investigaciones han establecido igualmente la importancia de los primeros años de vida como el período crítico en el 
cual es posible aplicar estimulación para lograr desarrollar el máximo potencial de cada ser humano.

De acuerdo a la teoría cognitivo-afectiva de Jean Piaget, el desarrollo de las funciones sensorio-motoras que ocurre durante los primeros años de vida influye posteriormente en el desarrollo de todos los procesos mentales; así, con ellas el niño aprende acerca de los objetos y las acciones, el espacio, el tiempo y la causalidad.

Para comprender esto, es necesario mencionar lo que plantean algunos autores, en el sentido de que todo el desarrollo del niño se resume en una corticalización progresiva, que está en función estrecha con las experiencias vividas. Así, si se actúa de manera positiva sobre un cerebro en proceso de maduración, aunque existan signos adversos que indiquen un pronóstico desfavorable, es posible observar que en todos los casos se realiza una lenta y progresiva maduración neurofisiológica que favorece la expresión espontánea del potencial básico remanente.

Según este modelo el niño está bajo la influencia de una gran cantidad de estímulos inespecíficos, de los cuales un pequeño porcentaje va a provocar la activación del sistema nervioso. El aprendizaje sensorio-motor de Piaget se basa en que durante el primer año de vida gran parte del aprendizaje del niño está dado por estímulos externos o sensaciones internas y una respuesta manifiesta, todas conductas motoras aprendidas. De acuerdo con esto, es de primordial atención la estimulación motriz y psicosocial en esta etapa.

Este sistema exige que el niño actúe en su medio para que el desarrollo cognoscitivo evolucione: el niño necesita moverse, manipular cosas, escuchar sonidos, mirar objetos para coordinar las acciones motoras simples con las percepciones que le van llegando (actos sensoriomotores). Su conocimiento es la construcción que éste realizará por sus acciones, descubriendo, creando o inventando, ayudado por la estimulación y el conocimiento social que se le brinda.

Entendemos entonces que el niño es producto de múltiples fuerzas (genéticas, psicológicas, sociológicas) y que su desarrollo recibe la influencia de factores internos y externos que actúan como un sistema dinámico y de desarrollo. Los factores intrauterinos brindan la estimulación necesaria para optimizar los resultados de la gestación, pero el parto prematuro ocasiona una alteración importante y repentina de este proceso ${ }^{(\underline{6})}$.

Debido a sus características peculiares $^{(\underline{7})}$, el recién nacido prematuro presenta dificultad para asimilar estímulos ambientales y menor organización interna, que se manifiesta en cambios de coloración de la piel, aumento del esfuerzo respiratorio, pobre regulación de la temperatura corporal e incapacidad para mantener un estado de alerta tranquila $(\underline{\underline{z})}$. Despiertan con llanto o reaccionan a cualquier estímulo a su alrededor demostrando escasa habilidad en los cambios de estado. Dichos signos afectan la capacidad del menor para interactuar con sus padres y con el medio ambiente, dedicando su esfuerzo sólo a la autorregulación (ㄱ).

Nuestra primera intervención estará orientada al niño, a los padres y a la relación entre ambos como aspecto importante para lograr cambios favorables en el desarrollo del menor. Los padres deben apoyar al recién nacido modulando su entorno y controlando estímulos para ayudarlo a mantenerse estable en la organización de su conducta (). 


\section{- Muestra}

Grupo de 46 pacientes lactantes hasta 2 meses edad (corregida) al ingreso; provenientes de las comunas del Servicio de Salud Concepción y del Servicio de Salud Arauco, seleccionados en dos grupos:

Grupo I: 24 RN con antecedente de prematurez, peso inferior a 1500 grs. y edad gestacional entre 26 y 30 semanas.

Grupo II: 22 RN con antecedente de prematurez, peso inferior a 1500 grs. y edad gestacional entre 31 y 37 semanas.

Estos niños fueron derivados de la unidad de Neonatología del Hospital Clínico Regional de Concepción, al momento de su alta o durante su hospitalización, razón por la cual ingresaron al programa durante el primer mes de edad corregida y algunos de ellos lo hicieron antes de cumplir las 40 semanas de gestación. Todos estos niños fueron ingresados para evaluación kinésica y de terapia ocupacional.

\section{- Intervención Terapia Ocupacional}

La estimulación sensoriomotora aplicada, al activar sistemas sensoriales importantes para la maduración, tiene como primer objetivo compensar la alteración de experiencias y permite auxiliar la reanudación de un proceso que se interrumpió de manera repentina con el parto prematuro ${ }^{(9)}$.

En la etapa de evaluación temprana de la conducta del recién nacido observamos su habilidad para interactuar con su entorno y su capacidad para tratar selectivamente estímulos ambientales, observar sus reacciones fisiológicas y motoras, así como su interacción o capacidad de alerta a estímulos visuales y/o auditivos, entregando a los padres indicaciones para proveer estimulación neurosensorial global ${ }^{(\underline{10})}$.

Durante las sesiones mensuales de estimulación se aplicaron las siguientes intervenciones:

- Entrevista a la familia, orientada a recoger antecedentes de salud general del menor y conocer estilos de manejo en el hogar;

- Observar nivel de actividad espontánea del niño y respuesta a estímulos del ambiente;

- Aplicar estimulación neurosensorial global de acuerdo a edad corregida.

- visual

- táctil

- auditiva

- vestibular

- estimular destrezas exploratorias y de juego

- lenguaje

- coordinación ojo mano 
- Entregar consejería a los padres sobre modalidades de estimulación del desarrollo psicomotor y manejo en el hogar de acuerdo a las necesidades específicas del menor.

- Reforzar la vinculación afectiva madre-hijo y favorecer la relación paterno-filial.

A los 6 meses de edad (corregida) se evaluó el desarrollo psicomotor de estos menores, para lo cual se utilizó la Escala de Evaluación del Desarrollo Psicomotor de Soledad Rodríguez (11), EEDP. Esta escala mide el rendimiento del niño frente a situaciones específicas que para ser resueltas requieren determinado grado de desarrollo psicomotor. Distingue cuatro áreas de funcionamiento específicas e independientes: área motora, área de lenguaje, área social y área de coordinación. Es la única pauta estandarizada para el país destinada a evaluar el desarrollo psicomotor de niños de 0 a 2 años. Los niños evaluados con esta pauta, y que presentan un desarrollo psicomotor de acuerdo a lo esperado para su edad, deben obtener un coeficiente cercano a 100. Así, con una desviación estándar hasta 85 se califica como Normal; un coeficiente entre 84 y 70 se califica como Riesgo y un coeficiente de 69 o menor se califica como Retraso del desarrollo psicomotor.

Si al efectuar dicha evaluación en los niños del programa se observa resultado de retraso, éstos son ingresados a tratamiento regular para nivelar su desarrollo, el cual se reevalúa a los 12 y a los 18 meses de edad (corregida). Estas reevaluaciones periódicas permiten aplicar estimulación precoz y oportuna adaptando la intervención a lo que se observa como necesidades y características individuales de cada niño. Este proceso se efectúa en estrecha colaboración con los padres, entregando en cada control instrucciones sobre técnicas y estilos de estimulación del desarrollo psicomotor, de acuerdo a edad.

\section{- Intervención Kinésica}

A su ingreso los niños fueron evaluados en su actividad refleja y su respuesta motora, utilizando al protocolo de evaluación del desarrollo evolutivo del Método Dinámico de Estimulación Kinésica (MEDEK) ${ }^{(12)}$.

Este método terapéutico permite influir y modificar positivamente el desarrollo evolutivo motor desde la más temprana edad. Utiliza estímulos específicos que van a provocar la activación del sistema nervioso obteniendo una máxima respuesta funcional del niño mediante la menor ayuda posible.

El método MEDEK sigue el criterio de la organización evolutiva de "modos neuromotrices", respetando los patrones del desarrollo ontogenético y considerando que se debe avanzar progresivamente en el tratamiento, sin saltar modos de conducta motora. La intervención terapéutica se inicia favoreciendo control de cabeza y luego en forma progresiva la sedestación, bipedestación y marcha, y por último se estimula la libre deambulación por medio de las integraciones superiores del equilibrio.

Con las técnicas del MEDEK no se trabaja sobre órganos o planos de integración del sistema neuromuscular individuales o aislados sino que por el contrario con cada ejercicio se pretende estimular la compleja interrelación funcional existente entre 
órgano laberíntico, ojos, receptores propioceptivos, músculos, tendones, ligamentos y superficies articulares $\underline{(12)}$.

Se espera que los menores intervenidos con este sistema logren los hitos del desarrollo motor en progresión céfalo caudal, de acuerdo a edad corregida.

En base a los resultados obtenidos en la evaluación inicial, se planificó el programa de ejercicios y maniobras requeridas en cada caso en particular, con 2 variantes:

a) el grupo de maniobras y ejercicios que debía ser aplicado por kinesióloga tratante.

b) El conjunto de recomendaciones terapéuticas basadas en el MEDEK que debían ser ejecutadas por los padres en el hogar, de acuerdo a un plan preestablecido y específico (repetición de los movimientos y maniobras por 10 veces, 3 veces al día).

Las sesiones aplicadas en kinesiterapia fueron de 30 minutos cada una, en las cuales se efectuó evaluación de progresos motores, control de las maniobras y ejercicios efectuados por los padres y entrega de indicaciones de refuerzo y/o progresión del programa de estimulación. Los resultados se tomaron y registraron a los 6 y 12 meses de edad (corregida) y se compararon con edad psicomotora según MEDEK.

\section{- Resultados}

\section{Evaluación de Terapia Ocupacional:}

a) Grupo de niños con edad gestacional de 26 a 30 semanas (Tabla 1a)

Doce niños (50\%) siempre estuvieron con desarrollo psicomotor dentro de límites normales. Los dos niños que obtienen resultado de Retraso a los 12 meses de edad corregida tienen antecedentes de leucomalacia (un caso) y embarazo múltiple (un caso). En este grupo etáreo se observan pesos al nacer entre los 658 y los 1360 grs. Del total del grupo, 5 niños presentaron asfixia leve a los 5 minutos de vida.

Tabla 1a. Evaluación de Terapia Ocupacional

Grupo de niños con edad gestacional de 26 a 30 semanas. $n=24$

\begin{tabular}{|c|c|c|c|c|}
\hline & \multicolumn{2}{|c|}{$\begin{array}{c}\text { EEDP a los } 6 \text { meses } \\
\text { de edad corregida } \\
n \quad \%\end{array}$} & \multicolumn{2}{|c|}{$\begin{array}{l}\text { EEDP a los } 12 \text { meses } \\
\text { de edad corregida } \\
\text { n } \%\end{array}$} \\
\hline Normal & 13 & 54.2 & 20 & 83.3 \\
\hline Riesgo & 9 & 37.5 & 2 & 8.3 \\
\hline Retraso & 2 & 8.3 & 2 & 8.3 \\
\hline
\end{tabular}


b) Grupo de niños con edad gestacional de 31 a 36 semanas (Tabla 1b)

Niño que persiste con retraso en su desarrollo psicomotor a los 12 meses de edad corregida presenta déficit sensorial (hipoacusia), hemorragia intracraneana grado I y antecedente de embarazo múltiple. En este grupo etáreo se observan pesos al nacer entre los 660 y 1650 gramos. Tres niños del grupo presentan antecedente de asfixia leve a los 5 minutos de vida.

Tabla 1b. Evaluación de Terapia Ocupacional

Grupo de niños con edad gestacional de 31 a 36 semanas. $n=22$

\begin{tabular}{|c|c|c|c|c|}
\hline & \multicolumn{2}{|c|}{$\begin{array}{c}\text { EEDP a los } 6 \text { meses } \\
\text { de edad corregida } \\
\mathrm{n} \quad \% \\
\end{array}$} & \multicolumn{2}{|c|}{$\begin{array}{c}\text { EEDP a los } 12 \text { meses } \\
\text { de edad corregida } \\
n \quad \% \\
\end{array}$} \\
\hline Normal & & 40.9 & 20 & 90.9 \\
\hline Riesgo & 10 & 45.5 & 1 & 4.5 \\
\hline Retraso & 3 & 13.6 & 1 & 4.5 \\
\hline
\end{tabular}

Evaluación Kinésica (Tablas 2a y $2 b$ )

Tabla 2a. Evaluación Kinésica

Grupo de niños con edad gestacional de 26 a 30 semanas. $n=24$

\begin{tabular}{|l|c|c|}
\hline & $\begin{array}{c}\text { EEDP a los } 6 \text { meses } \\
\text { de edad corregida } \\
\%\end{array}$ & $\begin{array}{c}\text { EEDP a los } 12 \text { meses } \\
\text { de edad corregida } \\
\%\end{array}$ \\
\hline Normal & 7.5 & 50 \\
\hline \hline Riesgo & 75.0 & 50 \\
\hline Retraso & 15.0 & \\
\hline
\end{tabular}

Tabla 2b. Evaluación Kinésica

Grupo de niños con edad gestacional de 31 a 36 semanas. $n=22$

\begin{tabular}{|l|c|c|}
\hline & $\begin{array}{c}\text { EEDP a los } 6 \text { meses } \\
\text { de edad corregida } \\
\%\end{array}$ & $\begin{array}{c}\text { EEDP a los } 12 \text { meses } \\
\text { de edad corregida } \\
\%\end{array}$ \\
\hline Normal & 4.5 & 57.2 \\
\hline \hline Riesgo & 75.0 & 33.3 \\
\hline \hline Retraso & 20.5 & 9.5 \\
\hline
\end{tabular}


* Retraso leve = hasta dos meses de retraso respecto a la edad cronológica

** Retraso moderado = hasta tres meses de retraso respecto a la edad cronológica

\section{- Discusión}

A la fecha han ingresado aproximadamente 140 niños al programa de seguimiento y estimulación de prematuros. Por diversas razones, un número importante de ellos abandona los controles o asiste en forma esporádica, siendo los motivos tanto económicos, como geográficos (alrededor de un $50 \%$ de niños son de procedencia rural, lo que implica traslados hasta de 4 horas para acceder al hospital), climáticos y de salud de los menores. La muestra analizada en el presente estudio estuvo constituída por aquellos niños que asistieron periódicamente a sus controles y fueron evaluados en las edades estipuladas.

De acuerdo a los resultados esperados podemos deducir que, en la evaluación kinésica a los 6 meses de edad corregida, en el grupo I el 75\% de los niños portadores de prematurez, bajo peso y nacidos entre 26 y 30 semanas de gestación, no obtuvo diferencias en su desarrollo psicomotor de más de 2 meses con respecto a edad cronológica según MEDEK. Es decir, el $75 \%$ de los niños logró control de tronco y sedestación regular, con equilibrio deficiente.

El 15\% de los niños resultó con retraso moderado, es decir, este porcentaje logró un completo control de cabeza a los 6 meses de edad corregida, pero sus reacciones de defensa (paracaídas) y de enderezamiento fueron logradas en forma regular y no lograron el control completo de tronco para la sedestación, sólo lo lograron con apoyo.

El 2,5\% de los niños resultó con retraso severo, logró solo un regular control de cabeza (un paciente con enfermedad respiratoria y poco estimulado).

El 7,5\% de los niños de este grupo resultaron sin retraso, es decir logró un completo control de cabeza, reacciones de defensa, enderezamiento y equilibrio y un completo control de tronco y sedestación.

El $82,5 \%$ logró el principal objetivo planteado.

En el grupo II, de un total de 44 niños prematuros, bajo peso y edad gestacional entre 31 y 37 semanas, a los 6 meses de edad corregida se observó que el $75 \%$ resultó con retraso leve, es decir no obtuvo diferencias en su desarrollo motor de más de 2 meses con respecto a edad cronológica según MEDEK. El 20,5\% resultó con retraso moderado. El 4,5\% resultaron normales, sin retraso, con las mismas condiciones que el grupo I. El 79,5\% logró objetivos planteados al inicio.

En la evaluación efectuada a los 12 meses de edad corregida, en el grupo I, el 100\% de los niños evaluados logró control de tronco y bipedestación, pero el $50 \%$ logró además marcha independiente (fluctuante en la población hasta el año 2 meses); por lo tanto se logró objetivo esperado. En el grupo II el 33,3\% de los niños logró control de tronco y bipedestación completa más marcha asistida. El 9,5\% logro control de 
tronco y bipedestación, sin marcha. E. 57,2\% logró control de tronco, bipedestación y marcha independiente.

De la observación sistemática efectuada a una amplia población de niños prematuros durante la aplicación del presente programa, se concluye que un número importante de ellos presenta dificultad en el seguimiento y atención visual, así como en atención auditiva, con retraso en el dominio de estas capacidades en relación a la población de niños nacidos de término, evaluados a la misma edad.

Aunque a primera vista los resultados obtenidos por las intervenciones kinésica y de terapia ocupacional pueden parecer contradictorias, en realidad ambas resultan concordantes. La evaluación kinésica muestra resultados exclusivamente del rendimiento motor del menor. La evaluación aplicada por terapia ocupacional muestra resultados de áreas motora, coordinación, social y lenguaje.

En la evaluación con EEDP para obtener el puntaje final del niño se suman todos los ítems respondidos exitosamente, y ello se traduce en que se obtenga en muchos menores evaluados un resultado general global de Normalidad, aunque se aprecien déficits en áreas específicas. Las áreas que reiteradamente aparecen como insuficientemente logradas y que condicionan el resultado de Riesgo o Retraso en la evaluación de terapia ocupacional son la motora y de lenguaje indicando que constituyen las de más difícil dominio en estos menores y coincidiendo con el resultado de la evaluación kinésica en el aspecto motor. Es necesario tener presente las limitaciones de la escala EEDP, la que evalúa el desempeño motor general sin considerar aspectos específicos del mismo, tales como reacciones de apoyo, giros de tronco, equilibrio o posición de cuatro pies.

Debido a los cuidados especiales que requieren, los niños prematuros pueden ver restringida su actividad global y observar limitaciones para interactuar con terceras personas más allá del núcleo familiar, lo que suele traducirse en insuficiente desarrollo del área social (fijación de la mirada, sonrisa social, mímica social). Constituyendo esta área un campo particularmente necesario de fortalecer, respetando los cuidados de salud que los menores necesiten.

A través de la frecuente interacción con los padres se observa, en aquellos que tienen su primer hijo, que parte importante de la información entregada por la profesional debe centrarse en cómo enfrentar ciertas conductas propias del comportamiento neonatal, e identificar en los menores señales indicadoras de la necesidad de apoyar el esfuerzo del recién nacido para autoregularse, modular el ambiente y mejorar su comunicación, lo que constituye un aspecto relevante para madres muy jóvenes o adolescentes. La intervención permitió entregar un conjunto amplio de habilidades a estas madres, orientada a facilitar el manejo en el hogar y la correcta estimulación de sus hijos.

De los resultados obtenidos en las evaluaciones es posible concluir la efectividad de las acciones aplicadas a este grupo, como una intervención necesaria y apropiada enfocada a disminuir los efectos negativos del nacimiento prematuro en el desarrollo psicomotor de estos menores. Así como deducir la utilidad de ampliar esta intervención a un mayor número de niños nacidos de pretérmino. Sin embargo, con el fin de profundizar este análisis, es indispensable puntualizar que: · existe un alto número de niños nacidos de pretérmino en el Hospital Clínico Regional de Concepción que por diversas razones no accedieron al programa, la gran mayoría de los cuales observa un 
desarrollo psicomotor adecuado a edad corregida, según se desprende de lo observado en controles médicos regulares con neonatólogo y neurólogo.

Existe un porcentaje de niños nacidos de pretérmino que por diversas razones no acceden a este programa, evaluados con retraso o riesgo de retraso del desarrollo psicomotor, ingresando en edades posteriores a tratamiento corrector regular en nuestro servicio. La edad promedio en que este grupo evidencia problemas es alrededor de 6-7 meses de edad corregida.

Dado que intervenciones como la propuesta implican un alto costo tanto para los servicios de salud (profesionales especializados, horas profesionales exclusivas, recintos adecuados) como para el niño y su familia (traslados, aumento de atenciones de salud, aumento de la carga de tareas hogareñas), la relación costo-beneficio de la misma es discutible.

Parecería entonces que la intervención temprana es útil y necesaria, pero no para todos los niños con antecedente de prematurez. La pregunta de a qué niños aplicar esta intervención debe ser materia de ulteriores estudios.

Aunque los resultados de la intervención se midieron a los 6 y a los 12 meses de edad corregida, estos niños continuaron en controles de kinesiterapia y terapia ocupacional hasta los 18 meses de edad corregida. En este período observamos que aunque el resultado general es bueno, de Normalidad en el desarrollo psicomotor, es posible apreciar en algunos de ellos discretos déficits en las áreas motora y de lenguaje. Ello induce a pensar cómo incidirán estos déficits en el desempeño posterior de estos niños, particularmente en el ámbito académico, dado lo que sobre este tema muestra la literatura $(\underline{13})$ y nuestra propia experiencia profesional de trabajo con menores preescolares y escolares, derivados al nuestro servicio con diagnósticos de disfunción motora gruesa y fina o dispraxia del desarrollo, y que presentan el antecedente de prematurez. 


\section{Referencias}

(1) Normas Técnicas para la Estimulación y Evaluación del Desarrollo Psicomotor del Menor de 6 años", Programa de Salud del Niño y del Adolescente, MINSAL, 1993.

(2) Hubner, M., Ramírez R, "Sobrevida, Viabilidad y Pronóstico del Prematuro". Revista Médica de Chile, 2002, 130: 931-938.

(3) Palisano R. "Use of chronological and adjusted age to compare motor development of healthy pret erm and fullterm infants". Developmental Medicine and Child Neurology, 1986; 28: 180-187..

(4) Orientaciones Técnicas para el Seguimiento del RN $<1500$ gr $0<32$ semanas al nacer", División de _Salud de las Personas, MINSAL, 1999..

(5) Avaria M. "Desarrollo Psicomotor". Revista Chilena de Pediatría, 1999, 70: 162-167. .

(6) Als H., Lawhon G, et al. Individualized developmental care for the very low-birth-weight preterm infant", JAMA 1994; 272: 853-858.

(7) Brazelton B. Y Nugent K. "Neonatal Behavioral Assesment Scale", 3er. Ed.,1997, Ed. Paidós, España..

(8) Case-Smith J., Butcher L. "Parents Report of Sensory responsiveness and temperament in preterm infants". The American Journal of Occupational Therapy, 1998, 52: 547-553..

(9) Anderson , J. "Intervención sensorial con niños de pretérmino en UCI neonatal", American Journal of Occupational Therapy, 1986..

(10) Buehler D., Als H. "Efectiveness of Individualized Developmental care for Low-Risk Preterm Infants: Behavioral and Electrophysiologic Evidence". Pediatrics, 1995, 96: 923-931..

(11) Rodríguez S., Arancibia U., Undurraga C. "Escala de Evaluación del Desarrollo Psicomotor de 0-24 meses" . 4a. Edición, Santiago de Chile, Ed. GALDOC, $1987 .$.

(12) "Curso sobre retardo mental", varios autores. Ediciones de la Comisión Permanente para la atención del Retardo Mental, Caracas, Venezuela, 1980.

(13) Foulder-Hughes Linda, Cooke Richard. "¿Do Mainstream Schoolchildren who were Born Preterm have Motor Problems?". British Journal of Occupational. 